\title{
Proposal of a Reference Framework for Manufacturing Systems Engineering
}

\author{
Gregor von Cieminski ${ }^{1}$, Marco Macchi ${ }^{2}$, Marco Garetti ${ }^{2}$, and \\ Hans-Peter Wiendahl ${ }^{1}$ \\ 'IFA, University of Hannover, Germany ${ }^{2}$ DIG, Politecnico di Milano, Italy, \\ marco.macchi@polimi.it
}

\begin{abstract}
The authors propose a "generic" reference framework to integrate modelling methods typically adopted to engineer a manufacturing system under a unifying "umbrella". These methods - from formal descriptive modelling to analytical modelling and simulation modelling methods - originate from different disciplines and serve different purposes.
\end{abstract}

\section{INTRODUCTION}

Manufacturing systems engineering is an intrinsically established process: starting from product specifications resulting from product design activities, quality, time and cost constraints imposed by enterprise corporate decisions and, eventually, physical constraints from existing plants, it encompasses process specification, system design and system evaluation to finally accomplish manufacturing system design solutions. Despite the fact that the majority of production engineers essentially agree on the steps laid out for this process model and utilise it accordingly, the authors deem that a "unifying" reference framework - comprising both engineering activities and related modelling methods - is missing. In fact, until now, only "particular" reference frameworks have been established in specific fields and with reference to specific engineering methods. Besides being frameworks with a specific scope, they also often deal with a single step of the manufacturing sys- 
tems engineering process only - consider, for example, the engineering process typically proposed to carry out manufacturing simulation projects.

A shared reference framework might be obtained, in the authors' opinion, through the complete specification of the two main elements involved in the engineering cycle, the manufacturing system itself and the modelling activities. In the paper, a reference architecture - in the remainder, the "Morphology" - will determine key characteristics of a generic industrial manufacturing system (IMS). A reference engineering methodology - the "Engineering Process" - will define the modelling activities to accomplish engineering solutions and the methods - and tools - required. The "Morphology" is inspired by the GERAM framework (IFIP, 1998). It inherits - and specialises in the manufacturing context - GERAM life cycle and view concepts. It extends GERAM with a thorough definition of the manufacturing system entity, subject of the engineering study. The "Engineering Process" is derived from methodologies established in the industrial engineering discipline (simulation (Carrie, 1988) and facility layout planning (Meller, Gau, 1996)). It integrates, as a unique methodology, the utilisation of descriptive modelling methods - to provide formal representations of system properties - and evaluation methods (analytical, algorithmic, heuristic or simulation methods) - for system performance calculation.

Section 2 presents the "Morphology of IMS" in its constituting elements. Section 3 outlines the "Engineering Process of IMS". The overall framework is presented in section 4 discussing its position with respect to GERAM and its functionality in the manufacturing system design process. Section 5 eventually outlines ongoing and future research activities.

2

\section{THE MORPHOLOGY OF INDUSTRIAL MANUFACTURING SYSTEMS}

\subsection{The Scope of the Morphology}

The "Morphology" is defined to guide the modeller to establish the nature of the manufacturing system that he or she would like to model. To this regard, manufacturing systems engineering is comparable to the widely accepted product design process and its adoption of morphological analysis. According to (Zwicky, 1948), the morphological analysis consists of two main steps. Firstly, the definition of the parameters that are the key characteristics of a product (system), and of all possible distinctions of these parameters. This allows a systematic description of the product (system). Sec- 
ondly, the possible or feasible combinations of different distinctions of different characteristics then represent the possible configurations of the product (system) under scrutiny. Transferred to the context of manufacturing systems engineering, the use of the morphological analysis is intended to facilitate a systematic definition of the characteristics of a manufacturing system that is to be engineered. It is proposed to help the modeller in the identification of those modelling methods that might be suitable for description, engineering or re-engineering activities of the manufacturing system itself.

\subsection{The Morphology Modelling Framework}

A morphological matrix of manufacturing systems is thus established in order to be able to distinguish between the different types of such systems (Fig. 1).

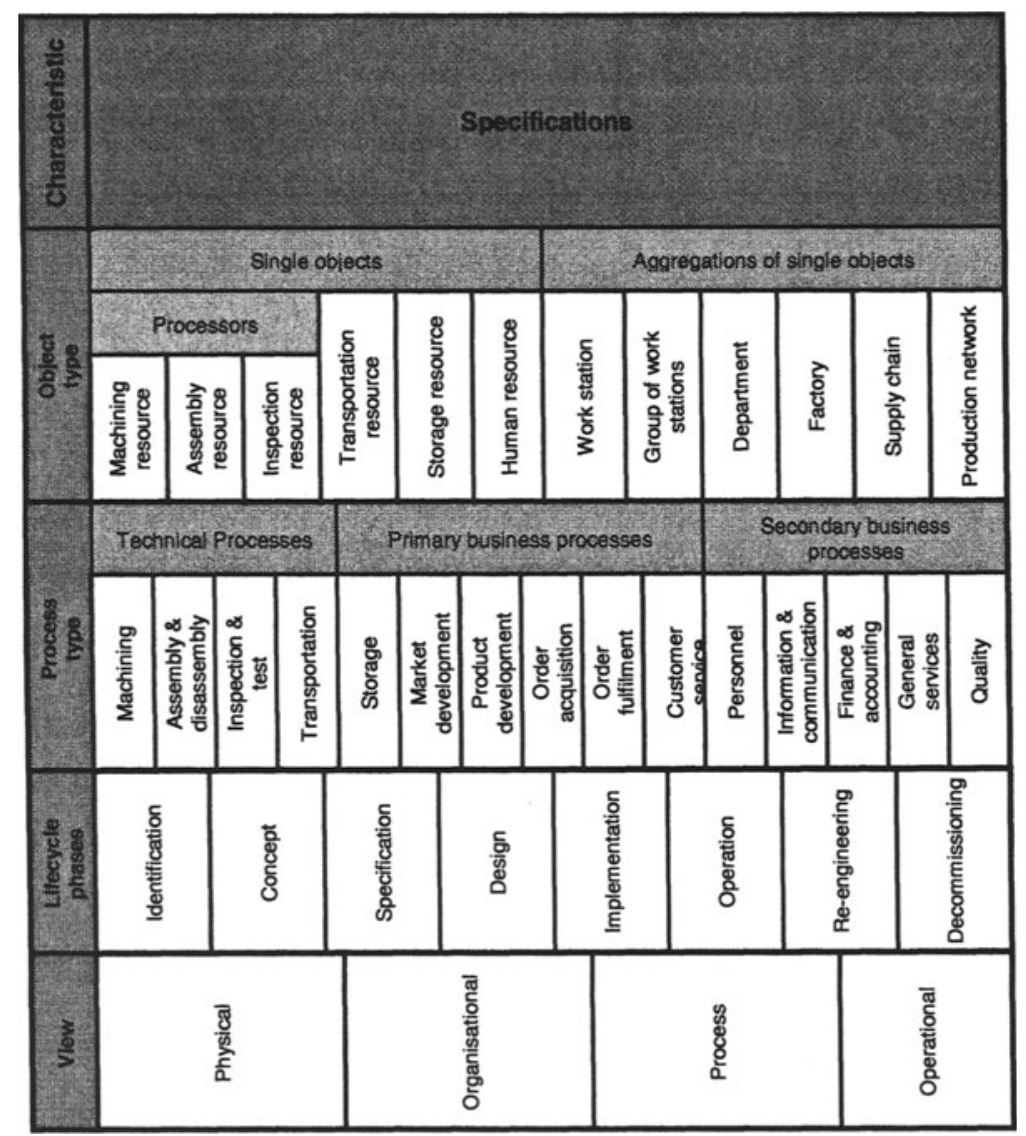

Figure 1: Morphological matrix for manufacturing systems 
The rows of the matrix correspond to the unique key characteristics that a manufacturing system possesses. Object and process type define, respectively, a system and a process taxonomy of a manufacturing system entity, whilst lifecycle phase and view specialise the GERAM lifecycle model and modelling views for a manufacturing system. For each of the four characteristics, one can distinguish between several specifications. All systems that exist can be described in detail by one combination of specifications of the four core characteristics.

The object type characteristic is concerned with the physical entities that represent a manufacturing system. All single objects encompass processing resources (machining, assembly and inspection processors), material handling resources and human resources. The aggregations of single objects are ordered according to accepted hierarchies of manufacturing systems. Starting from a workstation that might combine a machining, a human and a storage resource, the focus is widened until one arrives at the production network.

The process type characteristic defines three process types: technical processes, primary and secondary business processes. The definition of process types is inspired by similar models available in literature: compare, e.g., with CIMOSA definitions (CIMOSA, 1993), which group processes into manage processes (to develop business objectives and strategies and manage the overall behaviour of an organisation), operate processes (directly related to transformation of information and material into products) and support processes (providing assistance and maintenance to enterprise resources). The main scope of the "Morphology" are the operate processes (separated in technical processes and primary business processes) and the support processes (secondary business processes).

The lifecycle phase definition is inspired by lifecycle models proposed in enterprise engineering literature, in particular the GERAM life cycle model (IFIP, 1998) and comprise all phases of a manufacturing system.

The view characteristic specifies the modelling views that can be used to observe certain aspects that typically characterise a manufacturing system. Any manufacturing system is in fact characterised by four aspects: its physical structure, its production processes, the organisation of human resources and their relation with the physical structure and, finally, the co-ordination, control and support of its production processes. Thus, the physical view is concerned with the representation of all levels of physical aspects of a manufacturing system entity, from material flows to physical processes occurring during its operations. The organisational view focuses on the structure of responsibilities between the various human operators within a manufacturing system. The subjects of the process view are the "technological" processes, which set out the way that materials are transformed throughout the plant. 
The operational view, finally, refers to procedures to control and support the operation of the production processes.

\section{THE ENGINEERING PROCESS OF INDUSTRIAL MANUFACTURING SYSTEMS}

\subsection{Engineering methodologies in literature}

Up to now, "particular" methodologies have been developed for specific scopes. Amongst the methodologies developed around a specific method, the simulation methodology is a significant example. Simulation practitioners seem to agree on a common methodology consisting of a sequence of two main activities. A modelling activity aims at creating a "logical" (descriptive) model of the system (of its properties and behaviours (Vernadat, 1999)). A simulation activity then adopts a simulation model, created from system "logical" views, to evaluate the system behaviour (its expected performances) under experimental conditions. Amongst methodologies developed for specific applications, methodologies for facility layout planning are a significant example: the methodologies are built upon the final objective of the study, the determination of the physical organisation of production systems, and adopt proper methods for its accomplishment. A systematic layout design methodology is presented by Sly, et al (1997). Meller, Gau (1996) provide a comprehensive survey of the facility layout problem and methodologies and algorithms commonly adopted for its solution. Similarly to simulation, a common procedure is shared amongst practitioners consisting of "logical" modelling and performance evaluation activities. "Logical" modelling concerns the technological cycles (operations sequences/material flows), the plant structure and equipment (area requirements/constraints, equipment features) and the plant "design skeletons" (Sly, et al, 1996), where manufacturing "blocks" are assigned to material flows, laid out and graphically connected in a network-like fashion. The evaluation activity then seeks to analyse the performance of the "skeletons": e.g., distance-based evaluation methods are adopted to further detail "skeletons" through inter-"block" distances (Meller, Gau, 1996) and to evaluate performances either by means of algorithmic or heuristic models (Sly, et al, 1996).

The next section presents the proposal of a generic "Engineering Process of IMS", aiming to synthesise, in a "generic" methodology, existing "particular" models, such as those discussed in this section. 


\section{2 "Generic" engineering methodology}

The "Engineering Process" is structured as an IDEF0 model (Fig. 2). Five modelling activities are defined: Al concerns the identification of the production system organisation, automation level and control policies; A2 is concerned with the specification of the required technical processes; A3 concerns the specification of the system design solutions; A4 serves to develop the evaluation model and A5 concerns the evaluation of the system performance of the design solutions against performance objectives.

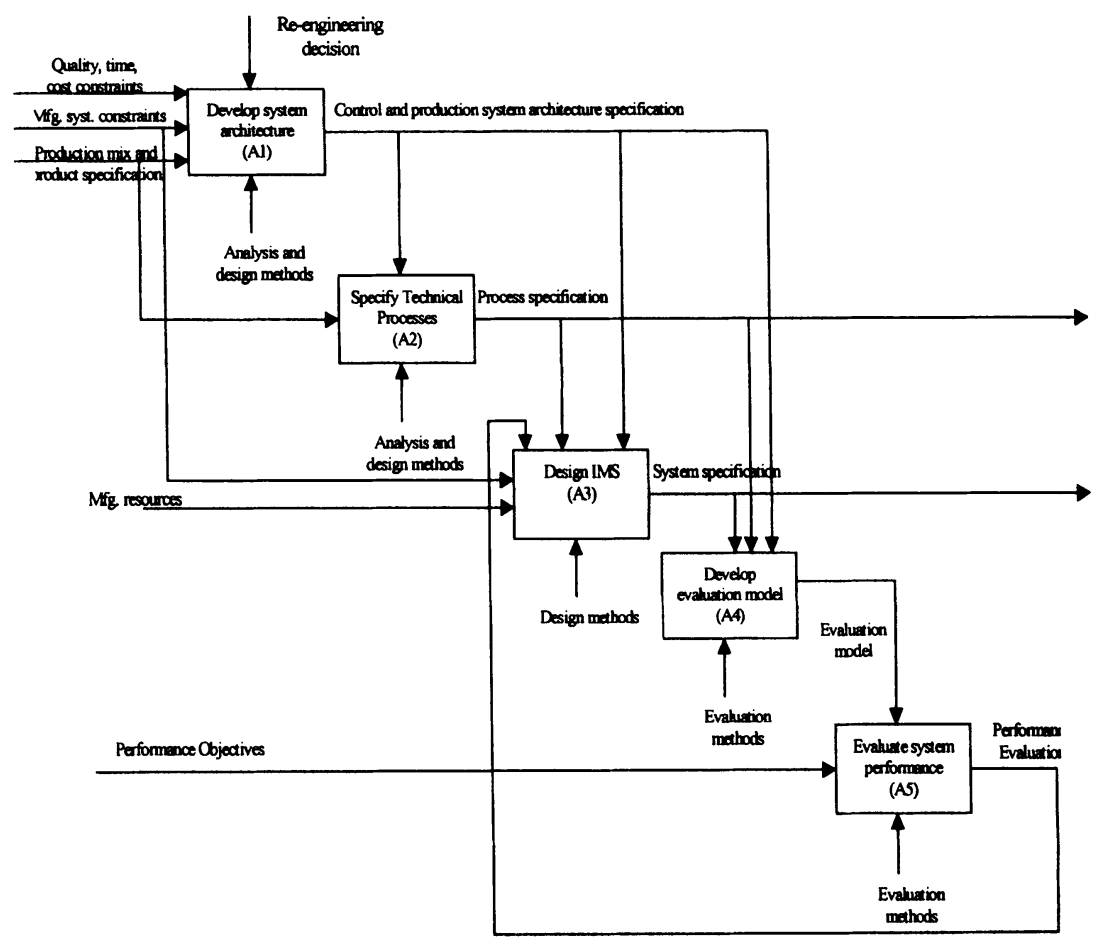

Figure 2: The generic engineering methodology - process model

The "best" solution results from one or more "evaluation" loops until performance objectives are achieved. The "best solution" is then utilised as an input to future implementation activities, both, as a process and system specification.

Having a closer insight, "develop system architecture" (A1) starts from given product designs and production mix (product bill of material, drawings and demand forecast), management decisions (cost, time, quality targets) and physical constraints (for existing plants) and sketches out a first draft of the design solution at architectural level. "Specify technical processes" (A2) is 
concerned with the specification of the production requirements (the process plans). "Design IMS" (A3) further details architectural drafts and defines a possible design solution capable to operate the "technical processes". It defines the allocation of manufacturing resources (equipment and human resources), their organisation (facility layout and human organisation), the processes and rules to operate and support the "technical processes". The final phases then seek to evaluate the design specifications. "Develop evaluation model" (A4) consists of translating the specifications into the ontology of the method adopted for evaluation. Hence, "evaluate system performance" (A5) represents the calculation of performance measures and the subsequent comparison with performance objectives by means of the evaluation model developed.

The methodology finally distinguishes amongst 3 types of modelling methods: analysis methods (for system analysis), design methods (for design specification), evaluation methods (for the evaluation of design specifications). The methods adopted by "particular" methodologies should be included in these types of methods. Referring, e.g., to facility layout planning, technological cycle diagrams are methods used to "specify technical processes" with an analytical scope. "Block" diagrams are adopted to set out draft layout designs. Distance-based methods are examples of evaluation methods.

\section{THE FRAMEWORK}

The framework proposed in this paper is concerned with the requirements and preliminary design phase of manufacturing system life cycle (Fig. 3). It defines a "generic" methodology to produce a manufacturing system design starting from its system concept. The methodology integrates analysis, design and performance evaluation methods. Being actually concerned with a generic definition, specific methods are not addressed. The authors foster integration of formal descriptive methods, derived from enterprise engineering and industrial engineering disciplines, and evaluation methods, typically adopted in industrial engineering (Garetti, Macchi, 2000). Whilst descriptive methods serve to produce formal views of the physical, operational, organisational and process aspects of manufacturing systems - thus serving analysis and design purposes -, evaluation methods integrate system views in a unique model for the sake of performance evaluation.

The engineering methodology is thus proposed as a unique basis for engineering method selection amongst all available methods. It should guide the modeller in selection either of suitable descriptive or evaluation methods competent to express models of all areas defined in the "Morphology". The 
"Morphology", in particular, is intended to guide the modeller in the selection of methods based on the life cycle phase and view concepts. Beside, the system and process taxonomy, facilitating the description of the "specific" configuration of the system under study, may further help in the selection whenever methods are "specific" to the system configuration itself.

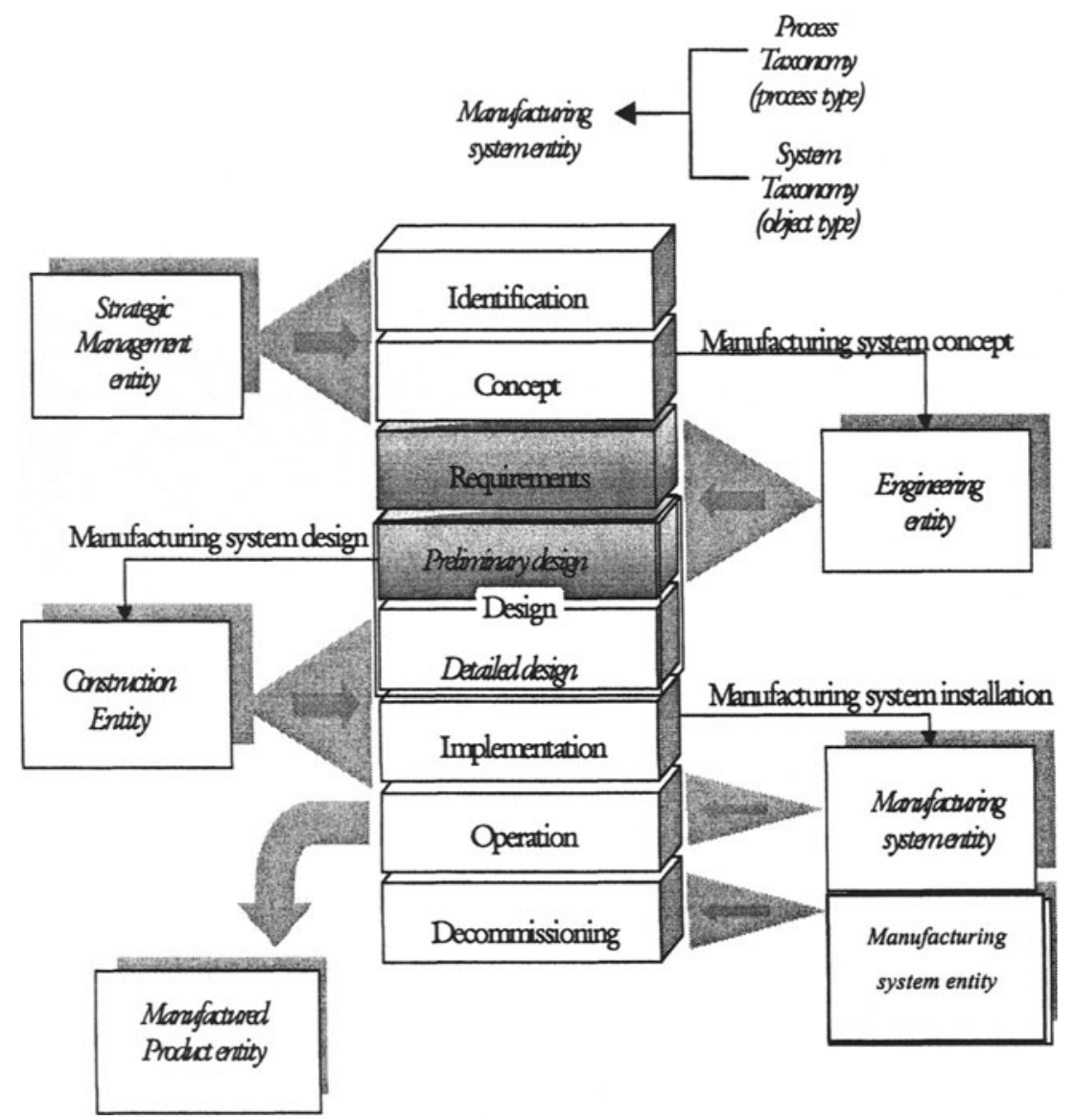

Figure 3: The Mfg. Systems Engineering framework and its relations with GERAM life cvcle

As an example of utilisation, the integration of methods for facility layout planning in the "Morphology" is shortly discussed. Their integration can be "generically" based on the life cycle phase and view concepts. Thus, e.g., technological cycle diagrams pertain to the requirements phase and process view. Block diagrams concern the design phase and physical view. Thereafter, referring to methods defined for "specific" system configurations, analytical models specifically built to calculate the throughput time of production lines, being either assembly, disassembly or machining lines, can be mentioned (Dallery et al., 1989, Di Mascolo et al., 1991). In the "Morphol- 
ogy", these methods should be classified as evaluation methods for specific process types - assembly, disassembly and machining processes - and object types - departments organised as lines - and subsequently selected.

\section{CONCLUSIONS}

In this paper, the authors' propose a framework that constitutes a unifying "umbrella" to be shared amongst both researchers and industrial practitioners. The framework represents a shared reference to integrate existing engineering methodologies. The proposal presented in this paper, however, is an initial proposition that is predominantly based on an abstract view of industrial engineering. Industrial case studies are envisaged that should provide a basis for comparison with more practical approaches to manufacturing systems engineering. In this way, the framework could be validated, especially in terms of a more "pragmatic" view, and extended by elements that are so far missing from an industrial point of view.

Future development work has then to validate the applicability of the manufacturing systems engineering approach proposed to the practical context in industry. The facility planning cycle has already been used as a basis for comparison in this paper. Wiendahl (1997) defines a systematic procedure that chiefly fulfils the practical requirements of real implementations. As is common practice in the industrial context, formal manufacturing modelling methods are not part of this practical development process.

Thus it has to be analysed what benefits a combination of this practical approach with the more theoretical approach presented in this paper holds. One unanswered question is whether this combination can help industry to effectively apply the formal modelling methods available in order to improve the design of manufacturing systems. Conversely, one ought to examine to what extent the confrontation with rather more "hands-on" approaches can lead academia to propose modelling methodologies that improve the planning quality and ease the planning efforts for practitioners.

The framework, and, in particular, the "Engineering Process of IMS", will be further developed to include specific modelling methods. In fact, methods, currently being reviewed within the VIMIMS project (see acknowledgements), will be classified in the "Engineering Process"-i.e. formal descriptive modelling methods such as IDEF0, UML and MES, analytical modelling methods such as Markov Chains, Fluid Markov Chains, Queuing networks, Petri-Nets and the Theory of Logistic Operating Curves, and discrete-event simulation methods. In VIMIMS, the framework will be used as a means to classify methods provided as educational contents. 
The results presented in this paper have been achieved as a side-activity of the VIMIMS project, an ongoing project funded by the European Union under action "MINERVA - Trans-national Projects in the Field of Open and Distance Learning (ODL) and Information and Communication Technology (ICT) in Education", whose aim is the establishment of a Virtual Institute for the Modelling of Industrial Manufacturing Systems (VIMIMS) for delivery of on-line courses to undergraduate students in production engineering disciplines. The authors thank other VIMIMS partners for collaboration and support: METID centre of Politecnico di Milano, Italy; LAG-INPG, France; SZTAKI, Hungary; ENFAPI Briantea, Italy.

\section{REFERENCES}

Carrie, A. (1988), Simulation of manufacturing systems. John Wiley \& Sons.

CIMOSA (1993), CIMOSA Open System Architecture for CIM, ESPRIT Consortium AMICE (Eds.), Springer-Verlag.

Dallery, Y., David, R. Xiao-Lan, X. (1989), Approximate analysis of transfer lines with unreliable machine and finite buffers. IEEE Transactions on Automatic Control, 34(9).

Di Mascolo, M., David, R. Dallery, Y. (1991), Modelling and analysis of assembly systems with unreliable machines and finite buffers. IIE Transactions, 23(4).

IFIP-IFAC Task Force, (1998), GERAM: Generalised Enterprise Reference Architecture and Methodology. Annex A to ISO 15704, ISO TC184/SC5/WG1 N423.

Garetti M. Macchi. M. (2000), Manufacturing systems modelling and enterprise modelling: do they need to be integrated? In: Proceedings of the Information Technology for Business Management ITBM - XXVI IFIP World Computer Congress WCC, Beijing, China.

Meller, R.D. Gau, K.-Y. (1996), The facility layout problem: recent and emerging trends and perspectives. Journal of Manufacturing Systems, vol. 15(5).

Sly, D.P., Grajo, E.S. Montreuil, B. (1996), Layout design and analysis software. IIE Solutions magazine, part 3 of a 3 part series.

Sly, D.P. (1997), Before dynamic simulation: systematic layout design from scratch. Proceedings of the Winter Simulation Conference.

Vernadat F. B. (1999), Requirements for simulation tools in Enterprise Engineering. $15^{\text {th }}$ Int. Conf. On CAD/CAM, Robotics and Factories of the Future, Aguas de Lindoia, SP, Brazil.

VIMIMS Consortium, (2000), Virtual Institute for the Modelling of Industrial Manufacturing Systems (VIMIMS). Proposal submitted to MINERVA action of European Commission.

Wiendahl, H.-P. (1997), Betriebsorganisation für Ingenieure (Business Management for Engineers). $4^{\text {th }}$ edition, Carl Hanser Verlag, Munich, Vienna.

Zwicky, F. (1948), The Morphological Method of Analysis and Construction. Courant, Anniversary Volume, pp. 461-470, Intersciences Publishing, New York. 